\title{
SASKATCHEWAN CHRISTMAS MAMMAL COUNT — 1990
}

\section{Compiled by WAYNE C. HARRIS, Box 414, Raymore, Saskatchewan. SOA 3J0}

A total of 65 counts were received this year, down 3 from last year, reporting 39 species, a record high number. Of interest in this year's report was the first count report of a Big Brown Bat. This occurrence, similar to last year's Little Brown Bat, was from Fort Qu'Appelle where Ron Hooper reported finding a freshly dead specimen at a local church. Also Feral Cat is in the list for the first time courtesy of "the scruffiest animal you ever saw" on the Birch Hills count. A Bobcat at Weyburn and a dead Pine Martin at Squaw Rapids were only the second records for the count though these species are regular but rare within the province. A hybrid Mule Deer $X$ White-tailed Deer at White Bear provided the first report for the count (see Blue Jay 47(4):228 for a detailed description from the same area).

Pronghorn Antelope were reported well to the north of their normal winter range at Scott and at Douglas Provincial Park, while the 270 at White Bear is an exceptional count for the north side of the South Saskatchewan River. At the other end of the range where large numbers are normal, Govenlock and Grasslands National Park, none were reported. Have they shifted northward for this winter? The count of 372 Mule Deer at the south end of the Frenchman River Valley on the Grasslands National Park count is the highest number ever reported from one area.

The most frequently reported species was the White-tailed Deer followed by Coyote, Red Fox and White-tailed Jackrabbit. Snowshoe Hares dropped in frequency considerably; are they declining or was the cold weather a factor with people doing less walking and therefore finding fewer?

For weather, coverage and participants please refer to the Christmas Bird Counts found elsewhere in this issue. Symbols found in the tables are as follows:

* identified by tracks with estimated numbers in parentheses

+ seen during count period but not on count day

$L$ active lodges or huts seen with numbers in parentheses

D fresh diggings seen

d freshly dead animals found

$S$ odour detected

$H$ heard with numbers in parentheses

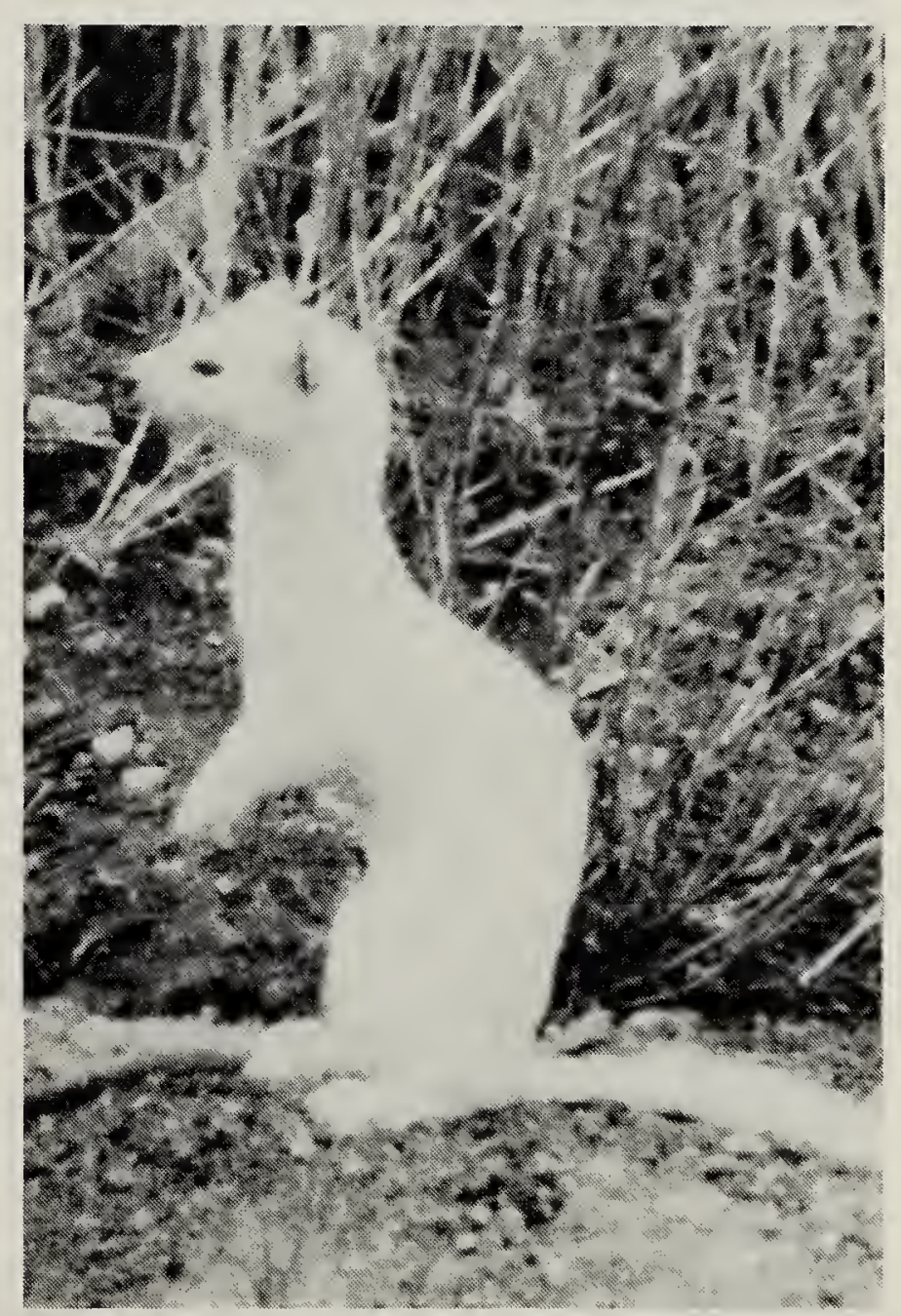

Long-tailed Weasel

F.A. Switzer 
Table 1-1. SASKATCHEWAN CHRISTMAS MAMMAL COUNTS

\section{SPECIES}

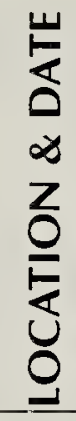

MASKED SHREW

SHREW SPECIES

BIG BROWN BAT

EASTERN COTTONTAIL

NUTTALL'S COTTONTAIL

SNOWSHOE HARE

WHITE-TAILED JACKRABBIT

RICHARDSON'S GR. SQUIRREL

GREY SQUIRREL

FOX SQUIRREL

RED SQUIRREL

NORTHERN FLYING SQUIRREL

NORTHERN POCKET GOPHER

BEAVER

DEER MOUSE

GAPPER'S RED-BACKED VOLE

MUSKRAT

MEADOW VOLE

VOLE SPECIES

MOUSE SPECIES

HOUSE MOUSE

PORCUPINE

COYOTE

WOLF

RED FOX

RACOON

FISHER

PINE MARTIN

ERMINE

LONG-TAILED WEASEL

LEAST WEASEL

WEASEL SPECIES

MINK

BADGER

STRIPED SKUNK

RIVER OTTER

BOBCAT

FERAL CAT

MULE DEER

WHITE-TAILED DEER

MULE X WHITE-TAILED DEER DEER SPECIES

MOOSE

ELK

PRONGHORN

TOTAL SPECIES

\begin{tabular}{|c|c|c|c|c|c|c|c|c|c|}
\hline & & & & & & & & & \\
\hline & & & & & & & 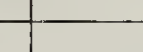 & & \\
\hline & & & & & & & & & \\
\hline & & & & & & & & & \\
\hline & & & & & 3 & & & & \\
\hline & & 4 & & 2 & 2 & & & *(10) & \\
\hline & 2 & 2 & ? & \begin{tabular}{|l|l|}
2 & $*(10$
\end{tabular} & )) & 7 & 5 & ${ }^{*}(3)$ & ${ }^{*}(7)$ \\
\hline & & & & & & & & & \\
\hline & & & & & & & & & \\
\hline & & & & & & & & & \\
\hline & & & & & & & & 2. & \\
\hline & & & & & & & & & \\
\hline & & ${ }^{*} * 1$ & & & & & & & \\
\hline & & & & & & & & & \\
\hline
\end{tabular}


Table 1-2. SASKATCHEWAN CHRISTMAS MAMMAL COUNTS

\begin{tabular}{|c|c|c|c|c|c|c|c|c|c|c|}
\hline 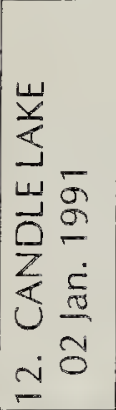 & 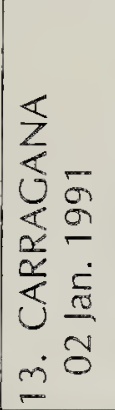 & 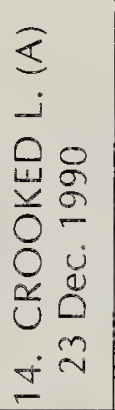 & 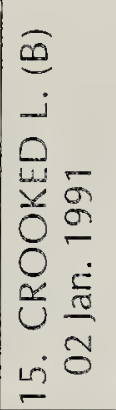 & $\begin{array}{l}\frac{0}{0} \\
0 \\
5 \\
0 \\
5 \\
0 \\
0 \\
0 \\
\infty \\
\infty \\
0\end{array}$ & 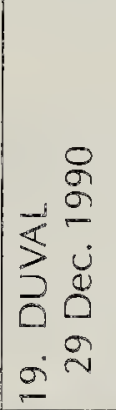 & 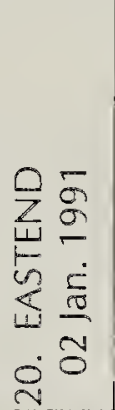 & 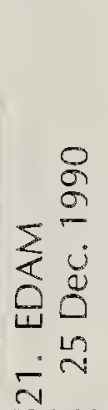 & 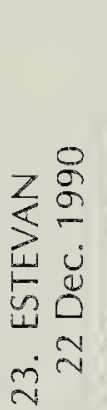 & 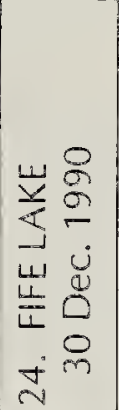 & 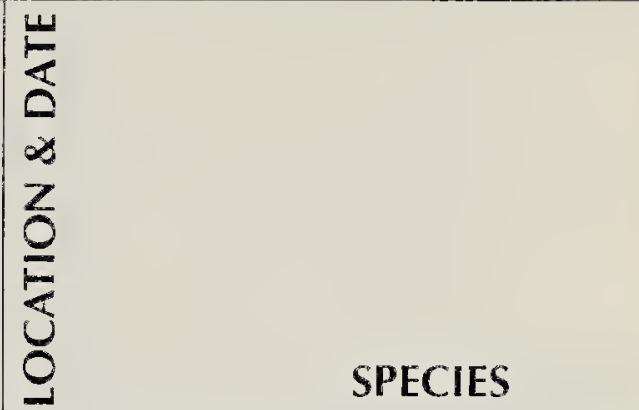 \\
\hline & & & & & & & & & & MASKED SHREW \\
\hline & & & & & & & & & & SHREW SPECIES \\
\hline & & & & & & & & & & BIG BROWN BAT \\
\hline & & & & & & & & 2 & & EASTERN COTTONTAIL \\
\hline & & & & 2 & ${ }^{*}(3)$ & & & $\mathrm{C}$ & 1 & NUTTALL'S COTTONTAIL \\
\hline & & $*(9)$ & * & 3 & $*(13)$ & & & & & SNOWSHOE HARE \\
\hline & & $*(2)$ & & & 1 & & & $*(9)$ & 1 & WHITE-TAILED JACKRABBIT \\
\hline & & & & & & & & & & RICHARDSON'S GR. SQUIRREL \\
\hline & & & & & & & & & & GREY SQUIRREL \\
\hline & & & & & & & & 2 & & FOX SQUIRREL \\
\hline 12 & 1 & 2. & & & & & & & & RED SQUIRREL \\
\hline & 1 & & & & & & & & & NORTHERN FLYING SQUIRREL \\
\hline & & & & & & & & & & NORTHERN POCKET GOPHER \\
\hline & & & & & & & & & & BEAVER \\
\hline & & & & & & & & & & DEER MOUSE \\
\hline & & & & & & & & & & GAPPER'S RED-BACKED VOLE \\
\hline & & & & & & & & & & MUSKRAT \\
\hline & & & & & & & & & & MEADOW VOLE \\
\hline & & & & & & & & & & VOLE SPECIES \\
\hline & & $*(5)$ & & & $*(4)$ & & & $*(3)$ & & MOUSE SPECIES \\
\hline & 3 & & & & & & & & & HOUSE MOUSE \\
\hline 1 & 1 & & & & & & 1 & 1 & & PORCUPINE \\
\hline 1 & & 1 & & 3 & ${ }^{*}(5)$ & 6 & 1 & $*(1)$ & 3 & COYOTE \\
\hline & & & & & & & & & & WOLF \\
\hline & 1 & & & 1 & $*(2)$ & 3 & & 2 & 1 & RED FOX \\
\hline & & & & & & & & & & RACOON \\
\hline & & & & & & & & & & FISHER \\
\hline & & & & & & & & & & PINE MARTIN \\
\hline & & & & & & & & & & ERMINE \\
\hline & & & & & $*(1)$ & & & & & LONG-TAILED WEASEL \\
\hline 1 & & & & & $*(1)$ & & & & & LEAST WEASEL \\
\hline & & & & & $*(1)$ & & + & & $*$ & WEASEL SPECIES \\
\hline & & & & & & & & & & MINK \\
\hline & & & & & & & & & & BADGER \\
\hline & & & & & & & & & & STRIPED SKUNK \\
\hline & & & & & & & & & & RIVER OTTER \\
\hline & & & & & & & & & & BOBCAT \\
\hline & & & & & & & & & & FERAL CAT \\
\hline & & & & 13 & & 26 & & & 16 & MULE DEER \\
\hline 19 & 3 & $*(2)$ & * & 6 & 8 & 42 & 17 & 6 & 30 & WHITE-TAILED DEER \\
\hline & & & & & & & & & & MULE X WHITE-TAILED DEER \\
\hline & & & & & & & & & & DEER SPECIES \\
\hline$*(1)$ & 1 & & & & & & & & & MOOSE \\
\hline 2 & & & & & & & & & & ELK \\
\hline & & & & 4 & & 16 & & & 6 & PRONGHORN \\
\hline 7 & 7 & 6 & 2 & 7 & 9 & 5 & 4 & 8 & 7 & TOTAL SPECIES \\
\hline
\end{tabular}


Table 1-3. SASKATCHEWAN CHRISTMAS MAMMAL COUNTS

\section{SPECIES}

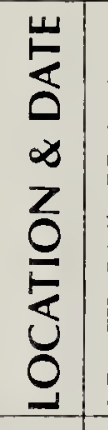

MASKED SHREW

SHREW SPECIES

BIG BROWN BAT

EASTERN COTTONTAIL

NUTTALL'S COTTONTAIL

SNOWSHOE HARE

WHITE-TAILED JACKRABBIT

RICHARDSON'S GR. SQUIRREL GREY SQUIRREL

FOX SQUIRREL

RED SQUIRREL

NORTHERN FLYING SQUIRREL

NORTHERN POCKET GOPHER

BEAVER

DEER MOUSE

GAPPER'S RED-BACKED VOLE

MUSKRAT

MEADOW VOLE

VOLE SPECIES

MOL ISF SFFCIFS

HOUSE MOUSE

PORCUPINE

COYOTE

WOLF

RED FOX

RACOON

FISHER

PINE MARTIN

ERMINE

LONG-TAILED WEASEL

LEAST WEASEL

WEASEL SPECIES

MINK

BADGER

STRIPED SKUNK

RIVER OTTER

BOBCAT

FERAL CAT

MULE DEER

WHITE-TAILED DEER

MULE X WHITE-TAILED DEER

DEER SPECIES

MOOSE

ELK

PRONGHORN

TOTAL SPECIES
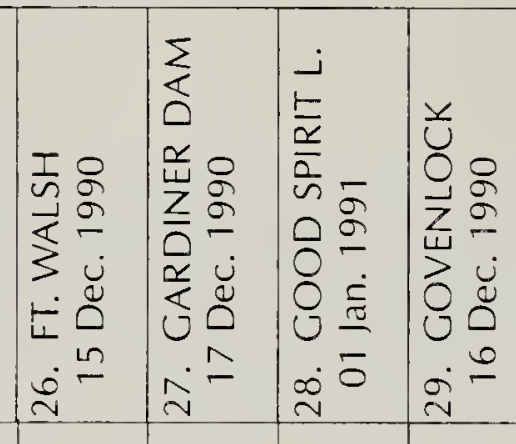

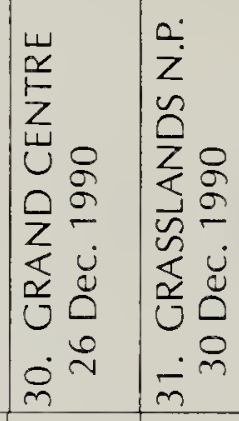

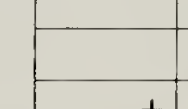

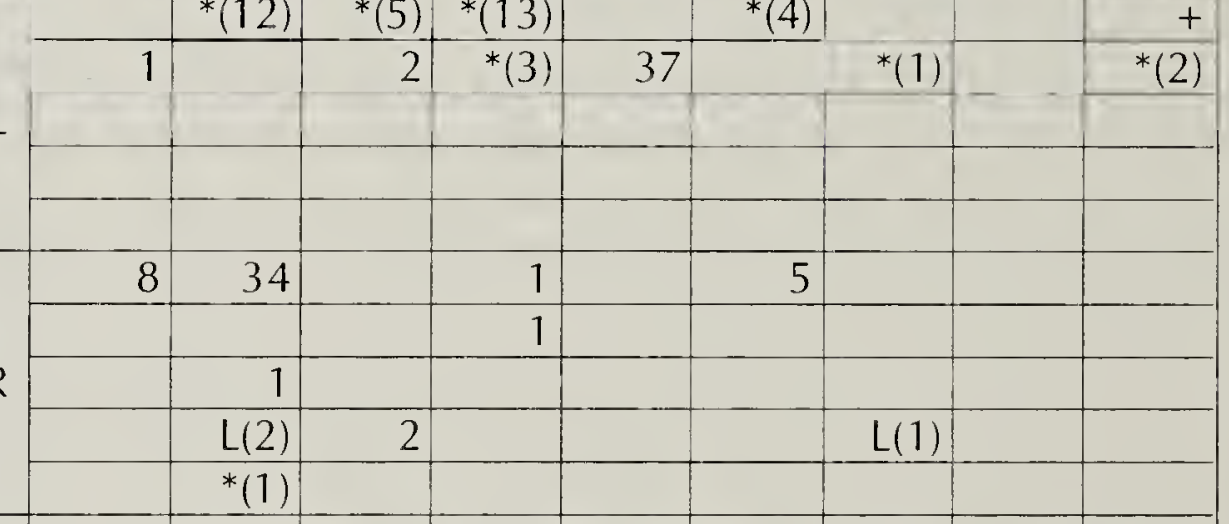

\begin{tabular}{|c|c|c|c|c|c|c|c|}
\hline & (1) & & (1) & & & & \\
\hline & *(15) & *(3) & & & & *(3) & \\
\hline+ & & & & & & & \\
\hline & 5 & $*(1)$ & 1 & & & 5 & \\
\hline 2 & 15 & 3 & 1 & 11 & $*(1)$ & 16 & 3 \\
\hline & & & & & & & \\
\hline 3 & 1 & ${ }^{*}(1)$ & & 2 & 1 & & 1 \\
\hline
\end{tabular}


Table 1-4. SASKATCHEWAN CHRISTMAS MAMMAL COUNTS

\begin{tabular}{|c|c|c|c|c|c|c|c|c|c|}
\hline 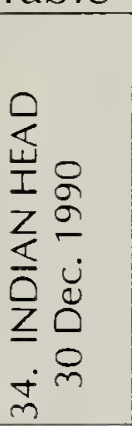 & 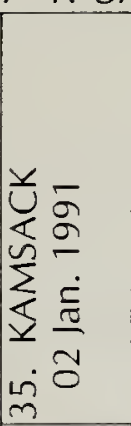 & 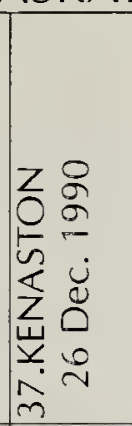 & 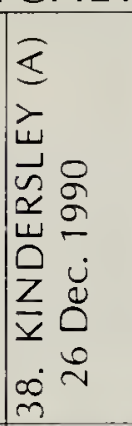 & 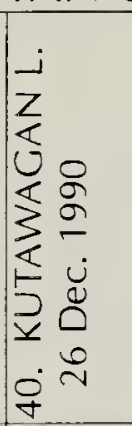 & 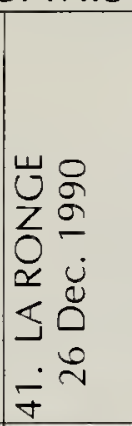 & 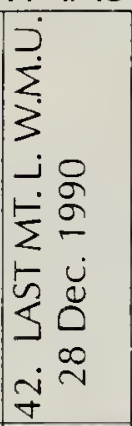 & 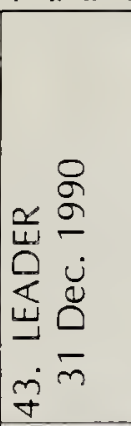 & 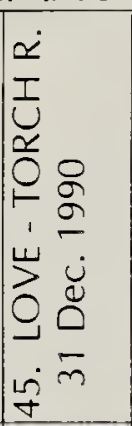 & 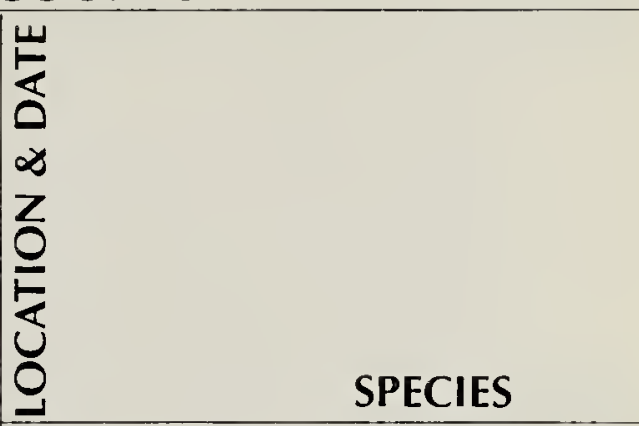 \\
\hline & & & & & & & & & \multirow{5}{*}{$\begin{array}{l}\text { MASKED SHREW } \\
\text { SHREW SPECIES } \\
\text { BIG BROWN BAT } \\
\text { EASTERN COTTONTAIL } \\
\text { NUTTALL'S COTTONTAIL }\end{array}$} \\
\hline & & & & & & & & & \\
\hline & & & & & & & & & \\
\hline & & & & & & & & & \\
\hline \multirow[t]{2}{*}{1} & & & & & & & & & \\
\hline & 17 & * & & $*(3)$ & $*(11)$ & $*(2)$ & & 1 & \multirow{5}{*}{$\begin{array}{l}\text { SNOWSHOE HARE } \\
\text { WHITE-TAILED JACKRABBIT } \\
\text { RICHARDSON'S GR. SQUIRREL } \\
\text { GREY SQUIRREL } \\
\text { FOX SQUIRREL }\end{array}$} \\
\hline 1 & & * & & 4 & & 4 & 2 & & \\
\hline & & & & & & & & & \\
\hline & & & & & & & & & \\
\hline \multirow{13}{*}{13} & & & & & & & & & \\
\hline & & & & & $*(3)$ & & $x^{2}$ & & \multirow{5}{*}{$\begin{array}{l}\text { RED SQUIRREL } \\
\text { NORTHERN FLYING SQUIRREL } \\
\text { NORTHERN POCKET GOPHER } \\
\text { BEAVER } \\
\text { DEER MOUSE }\end{array}$} \\
\hline & & & & & & & & + & \\
\hline & & & & & & & & & \\
\hline & & & & & & & & & \\
\hline & & * & & & & & & & \\
\hline & & & & & & & & & \multirow{5}{*}{$\begin{array}{l}\text { GAPPER'S RED-BACKED VOLE } \\
\text { MUSKRAT } \\
\text { MEADOW VOLE } \\
\text { VOLE SPECIES } \\
\text { MOUSE SPECIES }\end{array}$} \\
\hline & & & & & & & & & \\
\hline & & & & & $*(2)$ & & & & \\
\hline & & & & & & & 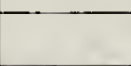 & & \\
\hline & & & & & & & 6 & & \\
\hline & & & & & & & & & HOUSE MOUSE \\
\hline & & 1 & & $*(1)$ & & & 3 & & PORCUPINE \\
\hline \multirow[t]{2}{*}{6} & 2 & * & + & 1 & & 2 & 5 & $\overline{1}$ & COYOTE \\
\hline & & & & & & & & & WOLF \\
\hline \multirow[t]{15}{*}{5} & 10 & * & + & $*(1)$ & $*(2)$ & 1 & 1 & $*(3)$ & RED FOX \\
\hline & & & & & & & & & \multirow{5}{*}{$\begin{array}{l}\text { RACOON } \\
\text { FISHER } \\
\text { PINE MARTIN } \\
\text { ERMINE } \\
\text { LONG-TAILED WEASEL }\end{array}$} \\
\hline & & & & & & & & & \\
\hline & & & & & & & & & \\
\hline & & & & & $*(4)$ & & & & \\
\hline & & * & & & & & & & \\
\hline & & * & & & & & & & \multirow{5}{*}{$\begin{array}{l}\text { LEAST WEASEL } \\
\text { WEASEL SPECIES } \\
\text { MINK } \\
\text { BADGER } \\
\text { STRIPED SKUNK }\end{array}$} \\
\hline & & & & & & & & + & \\
\hline & & & & & $*(1)$ & & & & \\
\hline & & & & $d(1)$ & & & & & \\
\hline & & & & & & & & & \\
\hline & & & & & & & & & RIVER OTTER \\
\hline & & & & & & & & & BOBCAT \\
\hline & & & & & & & & & FERAL CAT \\
\hline & & & + & & & & 75 & & MULE DEER \\
\hline \multirow[t]{6}{*}{25} & 15 & * & & 3 & $*(1)$ & 5 & 58 & 16 & WHITE-TAILED DEER \\
\hline & & & & & & & & & \multirow{5}{*}{$\begin{array}{l}\text { MULE X WHITE-TAILED DEER } \\
\text { DEER SPECIES } \\
\text { MOOSE } \\
\text { ELK } \\
\text { PRONGHORN }\end{array}$} \\
\hline & & & & & & & & & \\
\hline & $*$ & & & & & & & & \\
\hline & 7 & & & & & & & & \\
\hline & & & & & & & & & \\
\hline 6 & 6 & 9 & 3 & 7 & 7 & 5 & 7 & 6 & TOTAL SPECIES \\
\hline
\end{tabular}


Table 1-5. SASKATCHEWAN CHRISTMAS MAMMAL COUNTS

\section{SPECIES}

MASKED SHREW

SHREW SPECIES

BIG BROWN BAT

EASTERN COTTONTAIL

VUTTALL'S COTIONTAIL

SNOWSHOE HARE

WHITE-TAILED JACKRABBIT

RICHARDSON'S GR. SQUIRREL GREY SQUIRREL

FOX SQUIRREL

RED SQUIRREL

NORTHERN FLYING SQUIRREL NORTHERN POCKET GOPHER BEAVER

DEER MOUSE

GAPPER'S RED-BACKED VOLE MUSKRAT

MEADOW VOLE

VOLE SPECIES

MOUSE SPECIES

HOUSE MOUSE

PORCUPINE

COYOTE

WOLF

RED FOX

RACOON

FISHER

PINE MARTIN

ERMINE

LONG-TAILED WEASEL

LEAST WEASEL

WEASEL SPECIES

MINK

BADGER

STRIPED SKUINK

RIVER OTTER

BOBCAT

FERAL CAT

MULE DEER

WHITE-TAILED DEER

MULE X WHITE-TAILED DEER

DEER SPECIES

MOOSE

ELK

PRONGHORN

TOTAL SPECIES

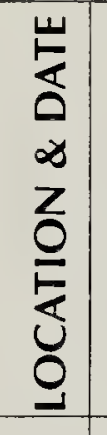

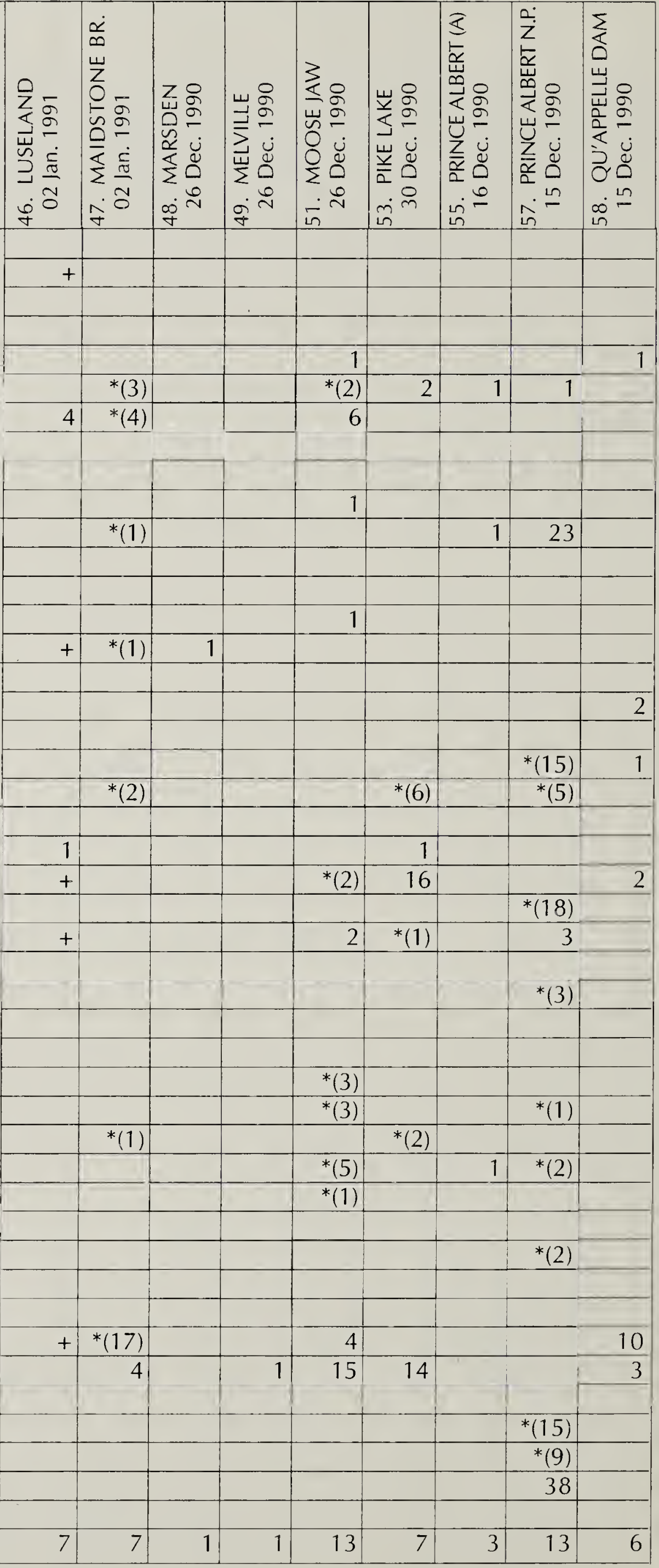


Table 1-6. SASKATCHEWAN CHRISTMAS MAMMAL COUNTS

\begin{tabular}{|c|c|c|c|c|c|c|c|c|c|}
\hline 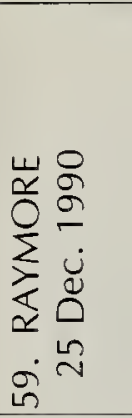 & 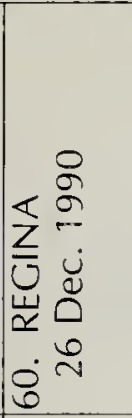 & 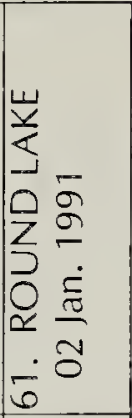 & 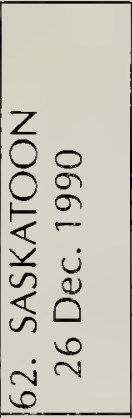 & 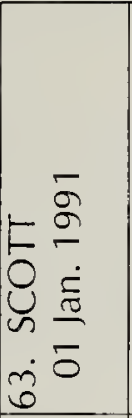 & 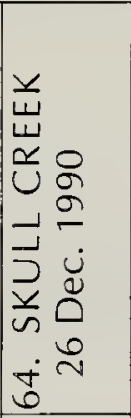 & 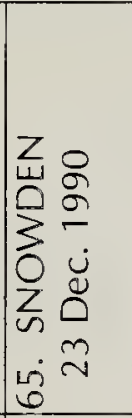 & 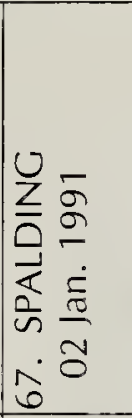 & 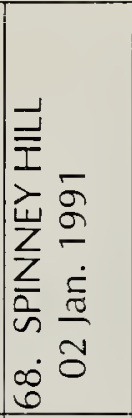 & 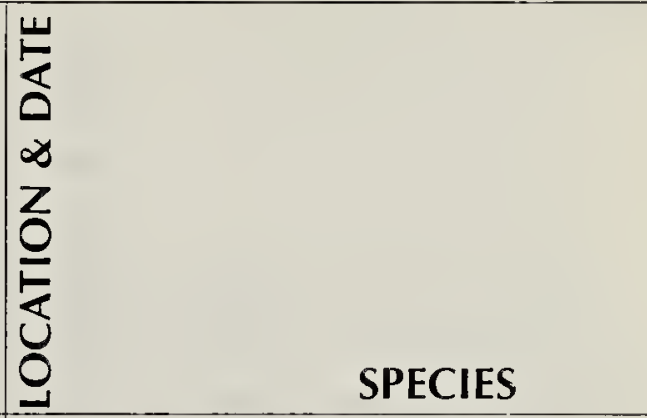 \\
\hline+ & & & & & & & $*(3)$ & 1 & \multirow{5}{*}{$\begin{array}{l}\text { MASKED SHREW } \\
\text { SHREW SPECIES } \\
\text { BIG BROWN BAT } \\
\text { EASTERN COTTONTAIL } \\
\text { NUTTALL'S COTTONTAIL }\end{array}$} \\
\hline$*(1)$ & & & & $*(2)$ & & & & & \\
\hline & & & & & & & & & \\
\hline & & & & & & & & & \\
\hline & & & & & $*$ & & & & \\
\hline$*(10)$ & & $*(5)$ & 1 & $*(10)$ & & 1 & * & $*$ & \multirow{5}{*}{$\begin{array}{l}\text { SNOWSHOE HARE } \\
\text { WHITE-TAILED JACKRABBIT } \\
\text { RICHARDSON'S GR. SQUIRREL } \\
\text { GREY SQUIRREL } \\
\text { FOX SQUIRREL }\end{array}$} \\
\hline 3 & 3 & 1 & 1 & ${ }^{*}(6)$ & * & $*(3)$ & & $*(2)$ & \\
\hline & & & & & & & & & \\
\hline & & & & & & & * & & \\
\hline & & & & & & & & & \\
\hline+ & & 1 & & & 4 & 1 & & 1 & \multirow{5}{*}{$\begin{array}{l}\text { RED SQUIRREL } \\
\text { NORTHERN FLYING SQUIRREL } \\
\text { NORTHERN POCKET GOPHER } \\
\text { BEAVER } \\
\text { DFFR MOUSF }\end{array}$} \\
\hline & & & & & & & & & \\
\hline & & & & & & & & & \\
\hline$L(1)$ & & & & & & & & & \\
\hline 1 & & & & & $*$ & & & ${ }^{*}(1)$ & \\
\hline+ & & & & & & & & & \multirow{5}{*}{$\begin{array}{l}\text { GAPPER'S RED-BACKED VOLE } \\
\text { MUSKRAT } \\
\text { MEADOW VOLE }\end{array}$} \\
\hline$L(3)$ & & & & & & & & & \\
\hline+ & & & & & $*$ & & & & \\
\hline & & & & & & $*(5)$ & & & \\
\hline & & & & ${ }^{*}(1)$ & & $*(9)$ & & & \\
\hline 4 & & & & & & & & & \multirow{5}{*}{$\begin{array}{l}\text { HOUSE MOUSE } \\
\text { PORCUPINE } \\
\text { COYOTE }\end{array}$} \\
\hline+ & & $*(1)$ & 1 & 1 & 2 & & & $*(1)$ & \\
\hline 3 & & 1 & 7 & 1 & 1 & ${ }^{*}(2)$ & $*$ & $*(2)$ & \\
\hline & & & & & & & & & \\
\hline 2 & 8 & $*(2)$ & 3 & 1 & 1 & + & $*$ & & \\
\hline & & & & & & & & & \multirow{5}{*}{$\begin{array}{l}\text { RACOON } \\
\text { FISHER } \\
\text { PINE MARTIN } \\
\text { ERMINE } \\
\text { LONG-TAILED WEASEL }\end{array}$} \\
\hline & & & & & & & & & \\
\hline & & & & & & & & & \\
\hline & & & & & & & & & \\
\hline & & $*(1)$ & 1 & & ${ }^{*}(4)$ & $*(2)$ & & & \\
\hline & & $*(1)$ & & & & & & & \multirow{5}{*}{$\begin{array}{l}\text { LEAST WEASEL } \\
\text { WEASEL SPECIES } \\
\text { MINK } \\
\text { BADGER } \\
\text { STRIPED SKUNK }\end{array}$} \\
\hline${ }^{*}(3)$ & & $*(1)$ & & & & $*(3)$ & $*$ & $*(1)$ & \\
\hline & & ${ }^{*}(1)$ & & & $*(2)$ & $*(1)$ & & & \\
\hline & & & & & & & & & \\
\hline & & & & $S(1)$ & & & & & \\
\hline & & & & & & & & & \multirow{5}{*}{$\begin{array}{l}\text { RIVER OTTER } \\
\text { BOBCAT } \\
\text { FERAL CAT } \\
\text { MULE DEER } \\
\text { WHITE-TAILED DEER }\end{array}$} \\
\hline & & & & & & & & & \\
\hline & & & & & & & & & \\
\hline & 3 & & & & 14 & & & & \\
\hline 3 & & 4 & 19 & ${ }^{*}(5)$ & 52 & 1 & $*$ & 8 & \\
\hline & & & & & & & & & \multirow{5}{*}{$\begin{array}{l}\text { MULE X WHITE-TAILED DEER } \\
\text { DEER SPECIES } \\
\text { MOOSE } \\
\text { ELK } \\
\text { PRONGHORN }\end{array}$} \\
\hline & & & & & & & & & \\
\hline & & & & & 1 & & & & \\
\hline & & & & & & & & & \\
\hline & & & & 3 & 220 & & & & \\
\hline 15 & 3 & 10 & 7 & 10 & 14 & 10 & 7 & 9 & TOTAL SPECIES \\
\hline
\end{tabular}


Table 1-7. SASKATCHEWAN CHRISTMAS MAMMAL COUNTS

\section{SPECIES}

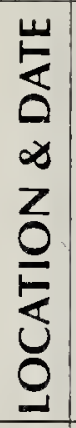

MASKED SHREW

SHREW Species

BIG BROWN BAT

EASTERN COTTONTAIL

NUTTALL'S COTTONTAIL

SNOWSHOE HARE

WHITE-TAILED JACKRABBIT

RICHARDSON'S GR. SQUIRREL

GREY SQUIRREL.

FOX SQUIRREL

RED SQUIRREL

NORTHERN FLYING SQUIRREL NORTHERN POCKET GOPHER BEAVER

DEER MOUSE

GAPPER'S RED-BACKED VOLE MUSKRAT

MEADOW VOLE

VOLE SPECIES

MOUSE SPECIES

HOUSE MOUSE

PORCUPINE

COYOTE

WOLF

RED FOX

RACOON

FISHER

PINE MARTIN

ERMINE

LONG-TAILED WEASEL

LEAST WEASEL

WEASEL SPECIES

MINK

BADGER

STRIPED SKUNK

RIVER OTTER

BOBCAT

FERAL CAT

MULE DEER

WHITE-TAILED DEER

MULE X WHITE-TAILED DEER

DEER SPECIES

MOOSE

ELK

PRONGHORN

TOTAL SPECIES
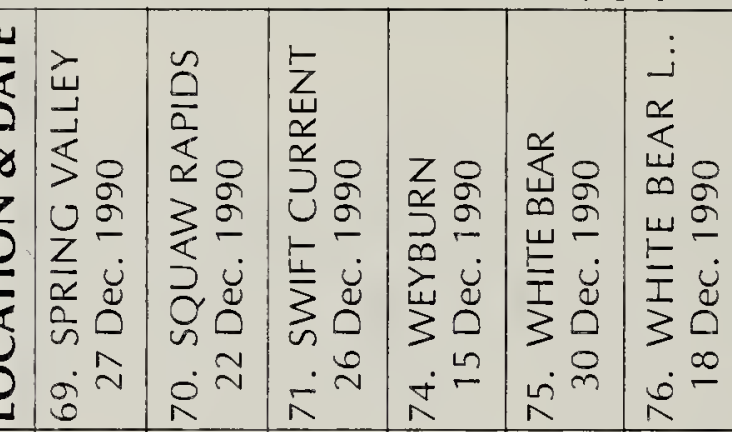

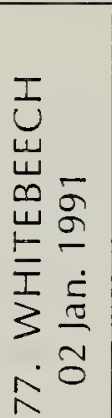

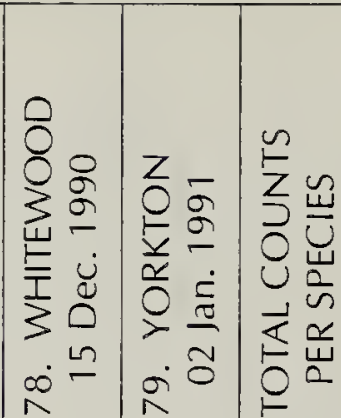

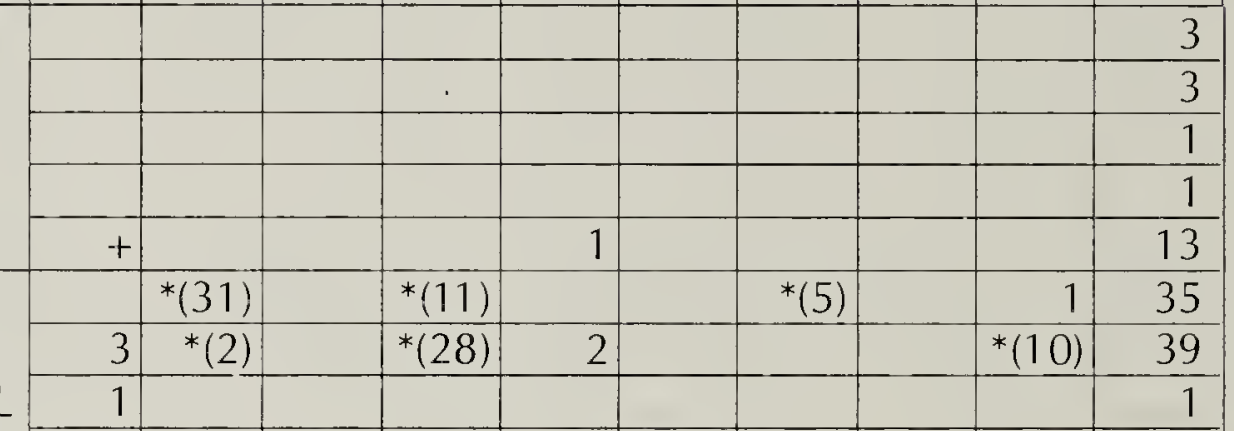

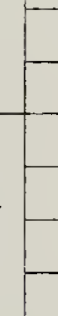

\begin{tabular}{|r|r|r|r|r|r|r|r|r|r|}
\hline & & & & & & & & & $\frac{1}{3}$ \\
\hline & & & 1 & & & & & & 3 \\
\hline & 10 & & & & 2 & 1 & & & 21 \\
\hline & & & & & & & & 3 \\
\hline & & & & & & & & 1 \\
\hline & $\mathrm{D}(2)$ & & & & & & & & 7 \\
\hline
\end{tabular}

\begin{tabular}{|c|c|c|c|c|c|c|c|c|c|}
\hline & $D(1)$ & & & & & & & & 3 \\
\hline & & & & & & & & & 3 \\
\hline$*$ & & & & & & & & & 6 \\
\hline * & $*(3)$ & & 1 & & & & & & 19 \\
\hline 5 & & & & & & & & & 4 \\
\hline to & 1 & & & + & & & & & 24 \\
\hline 2 & 7 & & 1 & 3 & & & & & 48 \\
\hline & & & & & & & & & 1 \\
\hline 1 & 1 & & 2 & 1 & & & & 1 & 45 \\
\hline 2 & & & & & & & & & 1 \\
\hline & & & & & & & & & 1 \\
\hline & $\mathrm{D}(1)$ & & & & & & & & 1 \\
\hline & & & & & & & & & 1 \\
\hline 1 & & & & & & & & & 10 \\
\hline & & & & & & & & & 7 \\
\hline & $\mathrm{D}(1)$ & & $*(1)$ & & & $*(1)$ & & $*(1)$ & 16 \\
\hline & $D(3)$ & & & & & & & $*(1)$ & 9 \\
\hline 2 & & & + & 1 & & & & & 6 \\
\hline & & & & & & & & & 3 \\
\hline & $*(1)$ & & & & & & & & 2 \\
\hline & & & 1 & & & & & & 1 \\
\hline & & & & & & & & & 1 \\
\hline+ & & 2 & & 140 & & & & & 23 \\
\hline+ & 1 & 3 & 4 & 4 & & $*(1)$ & 3 & $*$ & 55 \\
\hline & & & & 1 & & & & & 1 \\
\hline & & & & & & & & & 3 \\
\hline & 2 & & & & & & & & 6 \\
\hline & 7 & & & & & & & & 5 \\
\hline & & & & 270 & & & & & 9 \\
\hline 13 & 16 & 2 & 10 & 9 & 1 & 4 & 1 & 6 & \\
\hline
\end{tabular}

